\title{
Vaccination strategies against hepatitis A in Catalonia: vaccination coverage and impact on the disease
}

E. Borras $^{1}$, C. Requeijo ${ }^{2}$, G. Carmona ${ }^{3}$, L. Urbiztondo ${ }^{3}$, M. Martinez ${ }^{3}$, M. Jane ${ }^{1}$, C. Cabezas ${ }^{3}$

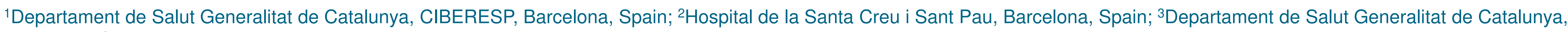
Barcelona, Spain

\section{Background}

Catalonia initiated a universal vaccination against hepatitis $A(H A)$ in 1999 in children between 11 and 12 years with combined hepatitis $A$ and $B$ vaccine. In 2014 vaccination strategy changed to a two-dose schedule in 2nd and 6th year of life. Second dose is scheduled after 5 years of the first dose (2019). Given the high effectiveness of a single dose of vaccine it is proposed to limit vaccination regimen to a single dose to assess vaccination coverage against $\mathrm{HA}$ in cohorts vaccinated according to the program and to estimate the impact of vaccination with $\mathrm{HA}$ on the disease.

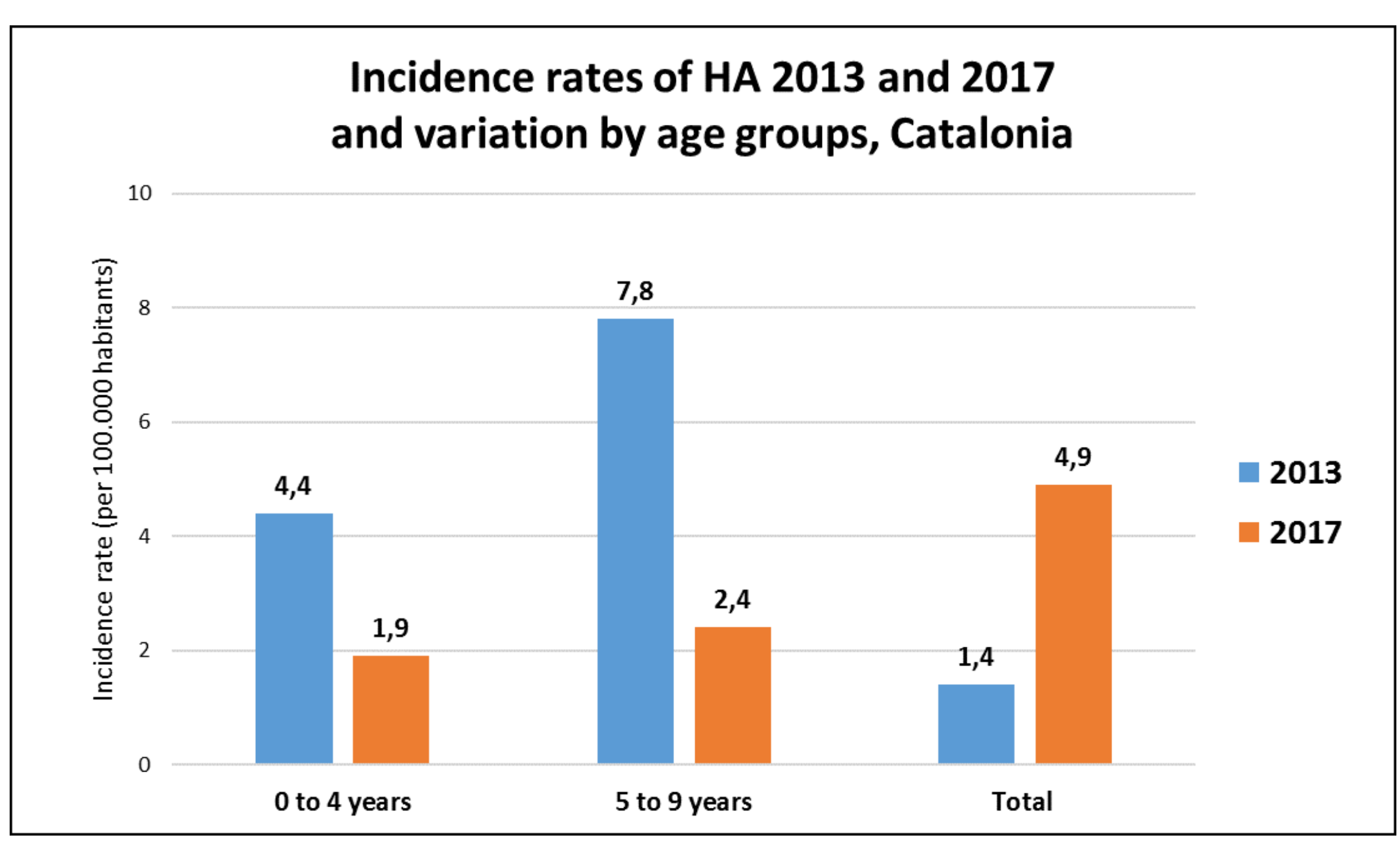

\section{Methods}

Vaccination coverage was obtained from the records of the Catalan Health Department. Incidence rate of hepatitis A was calculated using data from the infectious disease surveillance system of the Ministry of Health of Catalonia. Incidence rates in Catalonia (Spain) in the periods before (2013) and after (2017) were compared. The difference between the two periods was expressed as a percentage.

\section{Results}

The incidence rate of hepatitis $A$ in the 2013 period was 1.4 per 100,000 habitants, which up to 4.9 per 100,000 habitants in the 2017 period.

Incidence rate in children between $0-4$ years decreased by $56.8 \%$ and in children aged $5-9$ year by $69.23 \%$ (Table 1 ) in a context of increased global incidence. Vaccination coverage was higher than $85 \%$ in the 2 nd year of life and then $65 \%$ in the 6 th year of life.

\section{Conclusions}

$\checkmark$ There is a significant reduction in the incidence rate in cohorts vaccinated with a single dose, despite the context of increased global incidence caused mainly by an outbreak in men who have sex with men.

$\checkmark \quad$ It is necessary to assess vaccine effectiveness in order to change the vaccination strategy and reduce the schedule to a single dose. 